\title{
Beta-Band Oscillations without Pathways: the opposing Roles of D2 and D5 Receptors
}

\author{
Jean F. Liénard ${ }^{1,2 *}$ and Ignasi $\operatorname{Cos}^{1,2,3}$ and Benoît Girard ${ }^{1,2}$ \\ ${ }^{1}$ Institut des Systèmes Intelligents et de Robotique, Université Pierre \& Marie Curie, Paris, France \\ ${ }^{2}$ UMR 7222, CNRS, Paris, France \\ ${ }^{3}$ Universitat Pompeu Fabra, Center for Brain and Cognition, Barcelona, Catalonia, Spain \\ * jean.f.lienard@gmail.com
}

\section{Abstract}

Parkinson's disease is characterized by the death of dopaminergic neurons and the emergence of strong $\beta$-band oscillations throughout the basal ganglia nuclei. According to the mainstream theory, this synchrony is mediated by a dopamine deficit within the striatum creating a functional imbalance between the D1-expressing medium spiny neurons, which project to the internal segment of the globus pallidus, and D2-expressing one, which target its external segment, and ultimately leads to oscillatory activity. However, anatomical evidence gathered in rodents and primates has shown that striatal neurons are for the most part not organized into independent populations differentially targeting the two segments of the globus pallidus, nor alternatively expressing D1 or D2 receptors, thus calling for an alternative mechanism through which the lack of dopamine may cause oscillations. Here we adopt a computational approach in which we investigate a model whose parameters are fit to an extensive set of anatomical and physiological constraints from non-human primates, including axonal transmission delays gathered from eight experimental studies. Investigating the lack of dopamine in this model revealed that in the absence of segregated pathways, $\beta$-band oscillations emerge as a consequence of the extra-striate dopaminergic receptors reduced activity. These oscillations are caused by synchronous activity within the external globus pallidus-subthalamic nucleus loop, and their frequency are modulated by the transmission delays between these nuclei. Our model delivers a parsimonious explanation of oscillations that does not require any external driving influence from cortex, nor specific medium spiny neuron properties.

\section{Introduction}

Along with bradykinesia, hypokinesia, akinesia and resting tremor, one of the major hallmarks of Parkinson's Disease (PD) is the aberrant $\beta$-band oscillatory activity recorded in several nuclei of the Basal Ganglia (Marsden, 1984, 1989, Berardelli et al., 1996, Samii et al., 2004, Berardelli et al., 2001, Mazzoni et al., 2012). Specifically, stronger than normal $\beta$-band power $(18-35 \mathrm{~Hz})$ has been consistently revealed on EEG and MEG recordings from PD patients and in primate models of PD (Oswal et al., 2013), and is also found in electro-physiological recordings in the subthalamic nucleus (STN) and globus pallidus (GP) (Filion and Tremblay, 1991, Nini et al., 1995, Levy et al., 2000, Brown et al., 2001, Kühn et al., 2006, Weinberger et al., 2009). Remarkably, evidence that $\beta$-band activity can be restored to normal levels by administration of the DA precursor L-dopa (Brown and Marsden, 1999, Doyle et al., 2005), shows that DA and the intensity of neural activity in the $\beta$-band are intimately related (Oswal et al., 2013). However, the specifics of their relationship remain to be established.

Traditionally, a change in the balance between the direct and indirect pathways of the basal ganglia (BG) (Albin et al., 1989, Gurney et al., 2001, Frank et al., 2004, Frank, 2005) has been considered to be 
the origin of the anti-kinetic PD symptoms, which later extended to encompass the generation of abnormal oscillations (Humphries et al., 2006, Van Albada and Robinson, 2009, Van Albada et al., 2009, Tsirogiannis et al., 2010, Kumar et al., 2011, Lindahl and Hellgren Kotaleski, 2016). Yet frequent in the computational literature, mounting experimental evidence is gradually questioning this hypothesis. Specifically, to cause this imbalance, striatal medium spiny neurons (MSN) ought to be organized into independent populations expressing, exclusively, D1 or D2 receptors, which should project, respectively, to the internal and external segments of the globus pallidus (GPi/GPe). Although striatal pathways may be segregated in mice (e.g., Valjent et al. 2009, but see also Cazorla et al. 2014), tracing studies in monkeys (Parent et al., 1995, Lévesque and Parent, 2005) and rats (Kawaguchi et al., 1990, Wu et al., 2000, Fujiyama et al., 2011) have shown consistently that a majority of MSN projects both to the GPe and GPi. In addition, a variable proportion of MSN (up to 60\% in Nadjar et al., 2006) express simultaneously D1 and D2 receptors. Thus, at least for monkeys, and possibly for rats, there is serious anatomical evidence against these pathways acting and interacting separately.

Alternatively, stronger than normal $\beta$-band oscillations have also been linked to an imbalance of extrastriate DA receptors (Benazzouz et al., 2014), which are present across all basal ganglia nuclei (Rommelfanger and Wichmann, 2010) and are equally affected by DA loss. However, their roles remain to be fully understood. Former computational models have either disregarded them (Gurney et al., 2001, Gillies et al., 2002, Frank et al., 2004, Frank, 2005), or considered disparate parameter ranges for the temporal delays of axonal connections, which restricts the generality of their conclusions (Humphries et al., 2006, Leblois et al., 2006a, Van Albada and Robinson, 2009, Tsirogiannis et al., 2010, Nevado Holgado et al., 2010, Kumar et al., 2011, Lindahl and Hellgren Kotaleski, 2016). In this study we assess the role of extra-striate DA receptors in the basal ganglia oscillatory activity. To that end, we use a previously developed model of the macaque monkey basal ganglia that encompasses its neuro-anatomy, bio-physics, and neuro-physiology (Liénard and Girard, 2014, see Fig. 1). We extended this model to include realistic inter-nuclei neuro-transmission delays and we simulated the effect of a gradual DA depletion on the dynamics of the underlying neural circuitry. Our main result shows that as the level of DA transits below a critical level, the STN-GPe network starts oscillating within the $\beta$-band. The robustness and simplicity of our predictions strongly support that abnormal activity of extra-striate dopamine receptors are the likely cause of parkinsonian oscillations, and that their frequency is set by the axonal transmission delays of the STN-GPe loop.

\section{Methods}

\section{Our Model}

In this study we focus on the oscillatory activity of the BG circuitry at rest. With the exception of the changes described below (transmission delays optimized to fit to experimental studies and simulation of DA depletion on extra-striate receptors), the model that we present here adopts the same mathematical formalism and parameters we previously developed (Liénard and Girard, 2014). Briefly, each nucleus of the basal ganglia is simulated with a mean-field model incorporating the temporal dynamics of neurotransmitters, as in the initial version of this model (Liénard and Girard, 2014). These nuclei receive inputs from cortical (cortico-striatal neurons, CSN, and pyramidal tract neurons, PTN) as well as thalamic afferents (from the centromedian and the parafascicular nuclei, $\mathrm{CM} / \mathrm{Pf}$ ), which are implemented as independent random processes with different average firing rates (Bauswein et al., 1989, Turner and DeLong, 2000, Pasquereau and Turner, 2011, Pasquereau et al., 2015). As in Liénard and Girard (2014), the CSN input was simulated as a Gaussian process centered around $2 \mathrm{~Hz}, \mathrm{PTN}$ around $15 \mathrm{~Hz}$ and CM/Pf around $4 \mathrm{~Hz}$. The simulations presented here were obtained using a standard deviation of $2 \mathrm{~Hz}$, corresponding to a high noise in the neuronal activities. The oscillatory patterns obtained with this noise level were similar to those obtained with a lower standard deviation of $0.5 \mathrm{~Hz}$.

The parameter search of Liénard and Girard (2014) resulted in more than one thousand optimal model parametrizations that were equally maximizing the plausibility scores defined in that study. An additional assessment showed that the most of variability in these solutions is jitter of small magnitude $\left(<10^{-6}\right.$ in a 


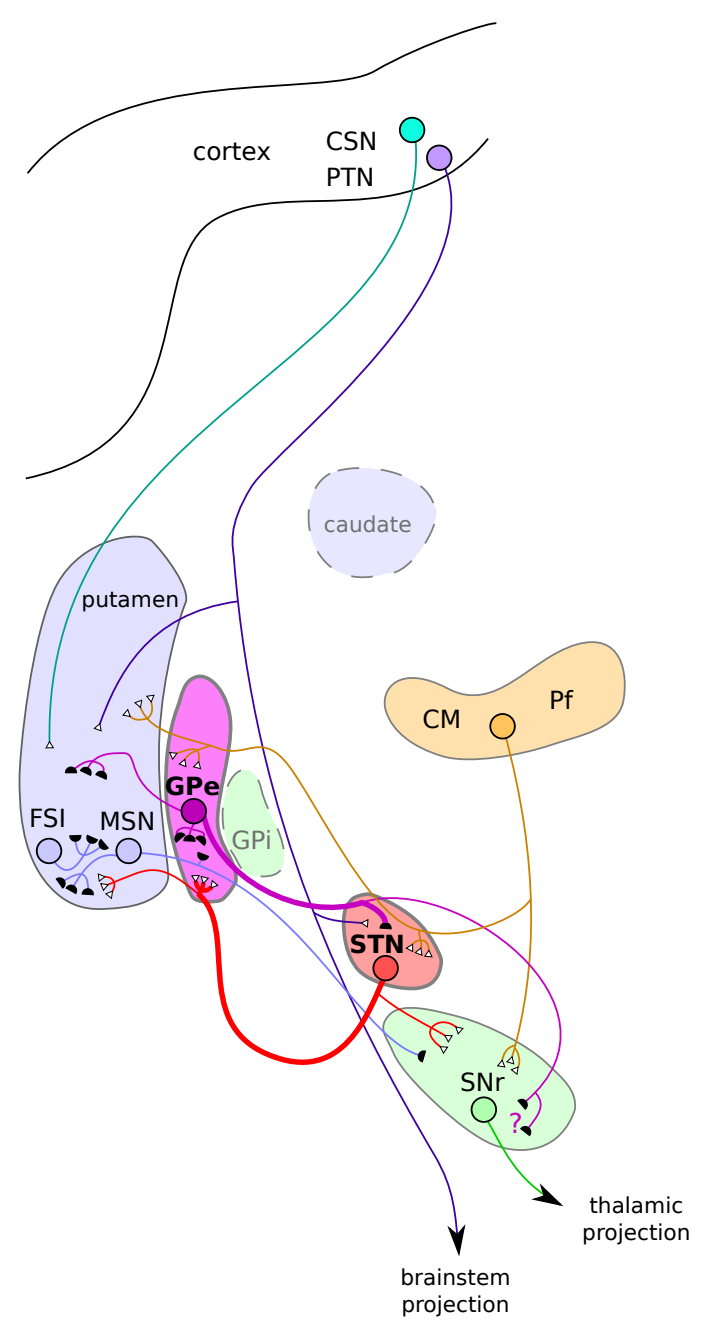

Figure 1: The nuclei of the basal ganglia and their interconnections. In this scheme, we do not assume the segregation of MSN on the basis to their D1 and D2 DA receptors. We show in this work that extra-striate DA imbalance can trigger the emergence of $\beta$-band oscillations in the GPe $\leftrightarrow$ STN loop. 
search space normalized within $[0,1])$ around 15 different base solutions. Thus we thus restrict our study to these 15 base solutions, as they globally represent the optimal parametrizations of the basal ganglia obtained in Liénard and Girard (2014).

\section{Transmission Delays in the Primate Basal Ganglia}

For our model to make sensible predictions about the naturally occurring frequencies of oscillation across basal ganglia nuclei, it was first required to establish the typical transmission delays across them for the case of the macaque brain. Unfortunately, the only data directly available to that end are not delays across nuclei, but rather delays between excitatory and inhibitory events recorded during stimulation studies (Yoshida et al., 1993, Turner and DeLong, 2000, Nambu et al., 2000, 2002, Kita et al., 2004, 2005, 2006, Tachibana et al., 2008, Iwamuro et al., 2009). Therefore, it was first necessary to engineer a methodology to properly identify the combinations of pathways and transmission delays involved in each experimental data set (see Tables S1 and S2), as to extract the specific delays across basal ganglia nuclei. First, we identified eight possible recurrent projections across all major nuclei: striatum, globus pallidus internal segment (GPi), globus pallidus external segment (GPe) and subthalamic nucleus (STN) (Fig. 2a). We scrupulously characterized each inter-nuclei projection (Fig. 2a), with the exception of the STN to Striatum and the GPe to Striatum as these connections are sparse (Jaeger and Kita, 2011, Liénard and Girard, 2014). Indeed, STN stimulation fails to elicit MSN activity (Kita et al., 2005); and although cortical stimulation elicits MSN overactivity, this is not followed by a noticeable second excitation which would have signaled an rebound mediated by the STN (Nambu et al., 2002). In addition, if the GPe to Striatum projection were functionally active in stimulation studies, we would expect the subsequent inhibition of the GPe to cause MSN overactivity, an event which has not been observed experimentally (Nambu et al., 2002). Finally, the anatomy reviewed by Liénard and Girard (2014) shows that these connections are structurally weak.

The method to estimate inter-nuclei delays consisted of comparing the inhibitions and excitations experimentally reported after stimulating the cortex, striatum, STN and GPe, with the timecourse of simulated activities resulting from combining different pathways in the basal ganglia circuitry (including axonal delays and neuronal integration time, see Computational Methods for further detail). To aid the optimization process, all delays were constrained to assume biologically plausible values (1-12 ms). Our exhaustive search to find the optimal fit thus implied the evaluation of all the $12^{8}$ ( $\approx 430$ million) possible combinations. Each combination was assigned a score dependent on the amount of experimental data it was able to replicate (see Supplementary Material for further detail).

\section{Extra-striate DA depletion}

Once the delays between nuclei were properly estimated (cf. previous section), we turned our attention to considerations on the distribution of extra-striate DA receptors, which have been identified across all BG nuclei (Rommelfanger and Wichmann, 2010). We hypothesized that an abnormal activation of extra-striatal DA receptors, combined with lagged activity due to inter-nuclei transmission delays, is the primary cause of $\beta$-band oscillations.

To test this hypothesis, we focused on accurately modeling the distribution of DA receptors within the GPe and STN only, as these are the only two nuclei which participate of multiple loops within the BG and could possibly cause oscillations. The GPi was disregarded, as it does not form any closed loop within the BG and cannot cause oscillations within the BG. Moreover, a review of the effects of varying DA concentrations on its activity reported contradictory data (Rommelfanger and Wichmann, 2010).

Since D2 receptors in GPe and STN are located at the pre-synaptic level only (Rommelfanger and Wichmann, 2010), we simulated their dynamics with an increase of the post-synaptic potential following an incoming spike, as described next: 


$$
\begin{aligned}
\mathrm{A}_{\mathrm{AMPA}}^{P D} & =\alpha \mathrm{A}_{\mathrm{AMPA}}^{\text {normal }} \\
\mathrm{A}_{\mathrm{NMDA}}^{P D} & =\alpha \mathrm{A}_{\mathrm{NMDA}}^{\text {normal }} \\
\mathrm{A}_{\mathrm{GABA}}^{P D} & =\alpha \mathrm{A}_{\mathrm{GABA}_{\mathrm{A}}}^{\text {normal }}
\end{aligned}
$$

where $\mathrm{A}_{\mathrm{AMPA}}, \mathrm{A}_{\mathrm{NMDA}}$ and $\mathrm{A}_{\mathrm{GABA}}$ are respectively the peak post-synaptic amplitude of a spike mediated

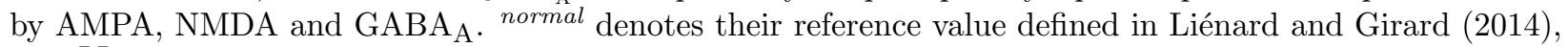
and $P D$ the increased level following DA depletion computed with the factor $\alpha$.

The STN D1-like receptors are of D5 sub-type, expressed at post-synaptic sites, and with constitutive activity (Chetrit et al., 2013). They have thus been modeled as modulators of the transfer function of the STN neuron population (see equation next), rather than as modulators of incoming activity:

$$
\theta_{S T N}^{P D}=\Delta_{\mathrm{STN}}+\theta_{S T N}^{\text {normal }}
$$

where $\theta_{S T N}$ is the average firing threshold of STN neurons, and $\Delta_{\text {STN }}$ is the offset created by DA depletion on D5 receptors.

Finally, based on the lack of projective selectivity of D1 and D2 MSN in macaque monkeys, we assumed that, on average, they compensate each other and that, consequently, their influence for the emergence of $\beta$-band oscillations is non-essential. This somewhat extreme modeling choice aims at studying to which extent the PD oscillatory phenomenon can be explained without segregated striatal pathways.

\section{Results}

\section{Transmission Delays in the primate Basal Ganglia}

The method used to calculate the delays between nuclei was based on exhaustive search, tailored to reproduce the timings recorded during stimulation experiments (see methods for further detail). This yielded the set of best fitting axonal delays shown in Table 1. To further ensure that the values obtained could be trusted, we performed an additional post-hoc fitness landscape inspection across the 8-D score space (Fig 2b). The fitness metric yielded a single peaked maximum along each dimension in the convex space of scores. Thus, the best fitting delays are globally optimal, and relaxing our constraint of maximum transmission delay beyond 12 ms would not increase the scores.

The top scoring delays obtained with this method were consistent with most (28 out of 30) of the experimental data considered and summarized in Tables S1 and S2. They exhibited some discrepancies with previous estimates, most notably for the subthalamo-pallidal interconnections (Table 1). While previous studies assumed that the $\mathrm{STN} \rightarrow \mathrm{GPe}$ and $\mathrm{GPe} \rightarrow \mathrm{STN}$ axonal delays to be similar, our systematic assessment revealed a substantial discrepancy between them (3 ms vs. $10 \mathrm{~ms}$, Table 1). An intuitive explanation of this timing imbalance may be obtained by looking at the timing of the early excitation of GPe following a cortical stimulation (recorded after roughly $9 \mathrm{~ms}$, cf. Table S2 and based on data from Nambu et al., 2000, Kita et al., 2004). The quick early excitation of GPe following cortical stimulations is most parsimoniously conveyed through the $\mathrm{Ctx} \rightarrow \mathrm{STN} \rightarrow \mathrm{GPe}$ pathway, given that the alternative pathway $\mathrm{Ctx} \rightarrow \mathrm{Str} \rightarrow \mathrm{GPe} \rightarrow \mathrm{STN}$ $\rightarrow$ GPe involves many more loops and is incompatible with other timings. Given that the Ctx $\rightarrow \mathrm{STN}$ connection has an axonal delay of about $6 \mathrm{~ms}$ (Nambu et al., 2000, Iwamuro et al., 2009), the STN $\rightarrow$ GPe connection delay has to be around $3 \mathrm{~ms}$. Intuitively, such a quick STN $\rightarrow$ GPe connection leads to a slow GPe $\rightarrow$ STN connection in order to satisfy the other timing constraints within the basal ganglia (Table S2 and $\mathrm{S1}$ ). Mechanistically, this imbalance in delays is consistent with the potential myelination of glutamatergic STN axons, which would result in an increased transmission velocity (Debanne et al., 2011). Corroborative evidence of the existence of STN axons myelination include its report in imaging studies made in monkeys (Yelnik and Percheron, 1979) and in rats (Koshimizu et al., 2013). Incidentally, a detailed computational study of STN neurons has found that their myelination may mediate the therapeutic effects of deep brain stimulation (Bellinger et al., 2008). 
bioRxiv preprint doi: https://doi.org/10.1101/161661; this version posted July 17,2017 . The copyright holder for this preprint (which was not certified by peer review) is the author/funder, who has granted bioRxiv a license to display the preprint in perpetuity. It is made available under aCC-BY 4.0 International license.

\begin{tabular}{lrrrrrrrrr}
\hline Connection & A & B & C & D & E & F & G & H & Ours \\
\hline $\mathrm{Ctx} \rightarrow$ Str & 6 & 2 & 4 & N/A & N/A & N/A & 2.5 & N/A & $\mathbf{7}$ \\
$\mathrm{Ctx} \rightarrow \mathrm{STN}$ & 5 & 1 & 1 & N/A & N/A & 5.5 & 2.5 & N/A & $\mathbf{3}$ \\
$\mathrm{Str} \rightarrow \mathrm{GPe}$ & N/A & 1 & 3 & N/A & N/A & N/A & 7 & N/A & $\mathbf{7}$ \\
$\mathrm{Str} \rightarrow \mathrm{GPi}$ & 10 & 1 & 3 & N/A & N/A & N/A & 7 & N/A & $\mathbf{1 1}$ \\
$\mathrm{STN} \rightarrow \mathrm{GPe}$ & N/A & 1 & 1 & 6 & 5 & 6 & 2 & 5 & $\mathbf{3}$ \\
$\mathrm{STN} \rightarrow \mathrm{GPi}$ & 5 & 1 & 1 & N/A & N/A & N/A & 4.5 & N/A & $\mathbf{3}$ \\
$\mathrm{GPe} \rightarrow \mathrm{STN}$ & N/A & 1 & 1 & 6 & 5 & 6 & 1 & 5 & $\mathbf{1 0}$ \\
$\mathrm{GPe} \rightarrow \mathrm{GPi}$ & N/A & 1 & 1 & N/A & N/A & N/A & 3 & N/A & $\mathbf{3}$ \\
\hline
\end{tabular}

$\overline{\mathrm{A}}$ :Leblois et al. (2006b) ${ }^{\mathrm{B}}$ :Van Albada and Robinson (2009) ${ }^{\mathrm{C}}$ :Tsirogiannis et al. (2010)

D:Holgado et al. (2010) ${ }^{\mathrm{E}}$ :Kumar et al. (2011) F:Pavlides et al. (2015) G:Lindahl and Hellgren Kotaleski (2016) ${ }_{\mathrm{H}}^{\mathrm{H}}$ :Shouno et al. (2017)

Table 1: Axonal delays in ms from previous computational studies of the primate BG and obtained with our model and fitting method.

The two experimental data to which the delays could not be fit to were in fact data that differed across experiments. Indeed, after cortical stimulation, Nambu et al. (2000) report a late STN excitation after 32.3 $\pm 11.8 \mathrm{~ms}$, while Iwamuro et al. (2009) report a $16.0 \pm 2.8 \mathrm{~ms}$ delay. These two numerical delays used in the optimization procedure are thus in contradiction. Our best fit to the whole set of data from the nine experimental studies predicted a $28 \mathrm{~ms}$ delay for the Cortical - STN stimulation, which is closer to the experimental data of Nambu et al. (2000). Similarly, the late excitation in GPe after a cortical stimulation occurs after $30.8 \pm 1.9 \mathrm{~ms}$ according to Kita et al. (2004) and $25.8 \pm 2.6 \mathrm{~ms}$ according to Nambu et al. (2000). Our best fit is a $32 \mathrm{~ms}$ delay, which favours Kita et al. (2004) over Nambu et al. (2000).

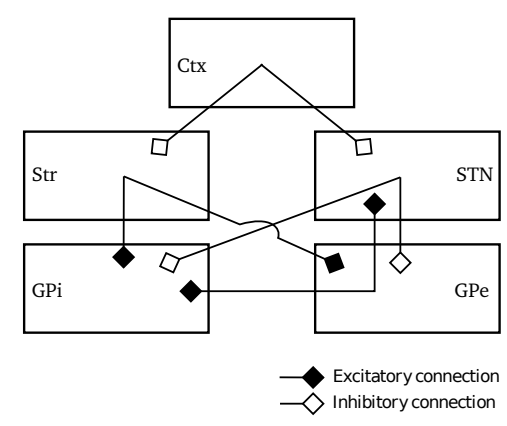

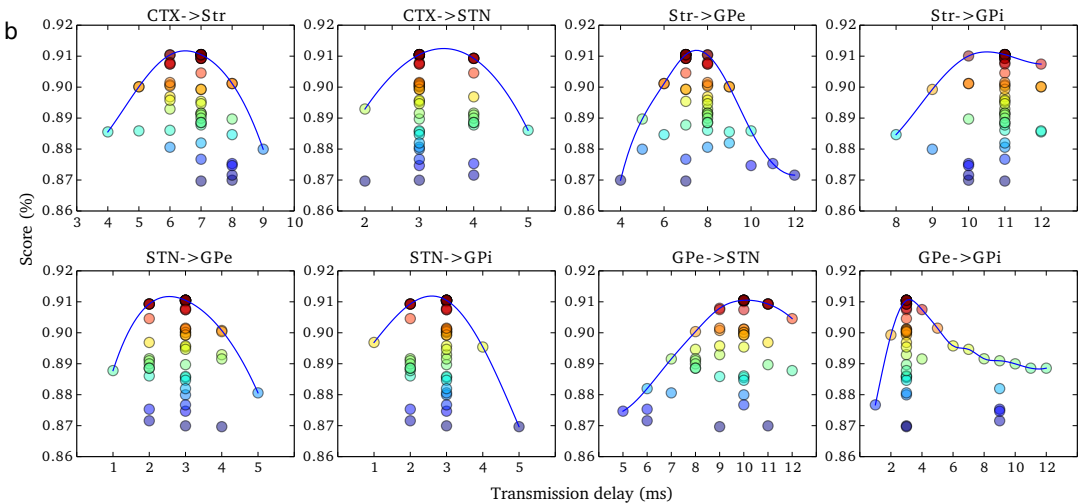

Figure 2: (a) Connections considered in the transmission delay estimation. (b) Slices in the score space of the transmission delay combinations, along each of the 8 dimensions (one panel per dimension). The $\mathrm{x}$-axis represents the delay (ms) of the set of delay combinations for the projection considered; its score is represented along the y-axis. We restricted the presentation to the best combinations of delays only, i.e., for each connection and each possible range of delay values for this connection, we only show the combination of delays with the best possible score (score $>0.86$, i.e. the maximal value of 0.91 minus 0.05 ). The best fitting solutions are shown by a dot, color coded according to its score (low-blue, high-red). The blue curves are cubic interpolations of the maximum scores for each delay value. Importantly, the score space seems to be single-peaked and globally convex in each dimension, implying the existence of a single maximum. 


\section{Distribution of $\beta$-Band oscillations in PD}

We simulated the effect of extra-striatal DA depletion on both the pre-synaptic D2-like receptors in GPe and STN and the post-synaptic D5 receptors in STN, while incorporating the axonal delays described in the previous section to our model. Our results show that an increase of the PSP amplitude in the GPe and STN and a moderate increase of the mean threshold between resting and firing rates in the STN generate an oscillatory regime in the $\beta$-band, with an average frequency of $28 \mathrm{~Hz}$ (See Eqs. 1 and 2, and Fig. 3A). In other words, the dynamics to generate oscillatory behaviour are consistent with a trade-off between the facilitation of the PSP, driven by the D2 receptors in STN and GPe, and the weakening of the excitability by the D5 receptors in STN (Fig. 4). In our simulations, oscillations between STN and GPe were phase locked, STN preceding GPe by $4 \mathrm{~ms}$ (see Fig. 3B).

It is worth noting that although our simulations also reported oscillatory activity following DA depletion in other nuclei such as the GPi and Striatum, including MSN and FSI cells (Fig. 4C), these oscillations did not originate locally. This was confirmed by the fact that setting the pallido-striatal and subthalamo-striatal connection activity to baseline cleared the striatal oscillatory activity. Furthermore, a deactivation of any of these projections also cleared the oscillations within FSI and MSN. By contrast, if the GPe $\rightarrow$ FSI connection remained active (see Figs. S5 and S6 in Supplementary Results), the oscillations re-emerged in the Striatum. Thus, oscillatory activity in the Striatum and GPi does not originate locally, but is conveyed from the phase-locked oscillatory activity of the GPe-STN loop.

As to reinforce our hypothesis of extra-striatal DA receptors being at the heart of the abnormally strong $\beta$-band oscillations, all fifteen model parametrizations considered in simulation (Liénard and Girard, 2014) reported the same frequency of oscillation when DA was depleted (Fig. 4). Furthermore, the frequency of oscillation depended on the projection delays only (Fig. 5), and its appearance on the alteration of connection strength and firing threshold (see Fig. 4).

\section{Discussion}

This study has shown that specific changes of bio-physical properties within the GPe-STN loop are sufficient to trigger $\beta$-band oscillations in the BG. A first prediction of the model is that, as the level of DA decreases, extra-striate D2 receptors gradually cause and intensify oscillations in the $\beta$-band, while D5 receptors weaken them. A second prediction is that decreasing the D5 receptor activity even further, by cancelling their remaining constitutive activity, would shift the dynamics of the system towards a steady state with no oscillations (Fig. 4, shift from the red to the blue area along the STN $\theta$ offset axis). This is consistent with observations by Chetrit et al. (2013), who showed that diminishing the D5 receptor constitutive activity in the STN of 6-OHDA PD rats did cancel abnormal neuronal activity and reversed motor impairment. Finally, oscillatory activity reported in other nuclei within the BG, e.g. FSI-MSN circuitry, does not originate locally, but is rather conveyed by axons from the GPe/STN loop.

These predictions resulted from extensive tests with a computational model of the macaque monkey basal ganglia, fitted from over a hundred independent anatomical and physiological experimental data in macaque monkeys (Liénard and Girard, 2014). Furthermore, to ascertain the delays between BG nuclei we also incorporated a set of stimulation studies to our calculations (Yoshida et al., 1993, Turner and DeLong, 2000, Nambu et al., 2000, 2002, Kita et al., 2004, 2005, 2006, Tachibana et al., 2008, Iwamuro et al., 2009) see section 2. Although this model contains numerous parameters, it is important to notice that these were optimized to fit to healthy non-human primate references. By contrast, the results and predictions of this study stem from varying two free parameters only, which model the process of DA depletion in GPe and STN: the D2 PSP amplification and D5 firing threshold increase.

\section{Axonal delays}

A vast number of stimulation studies aimed at characterizing transmission delays across different neuronal groups (Yoshida et al., 1993, Turner and DeLong, 2000, Nambu et al., 2000, 2002, Kita et al., 2004, 2005, 
A.

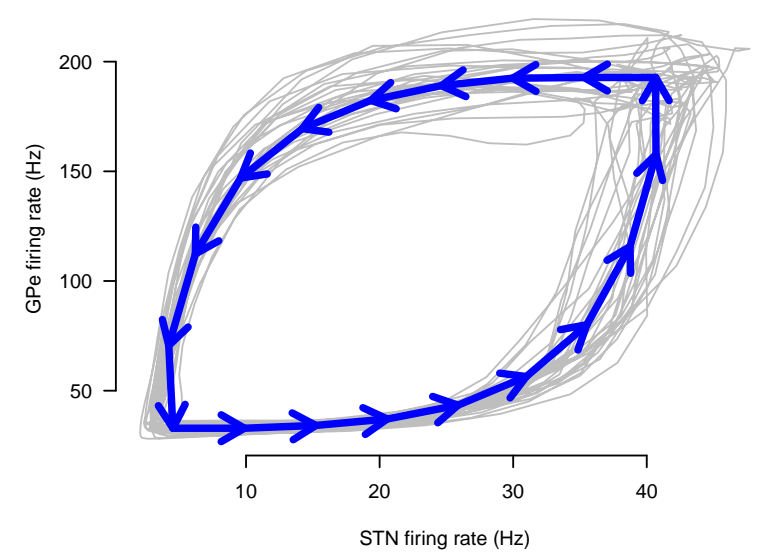

c.

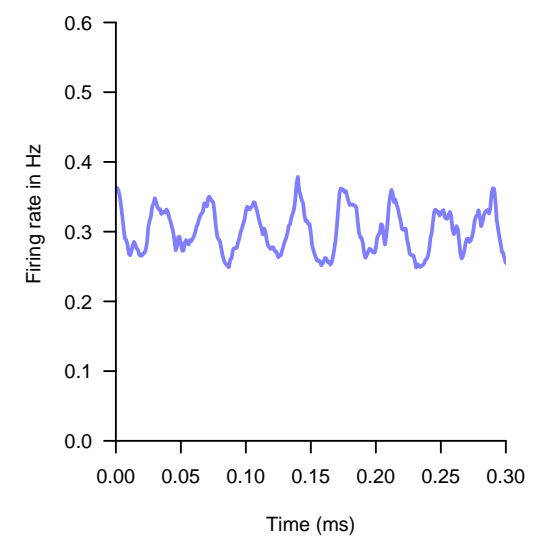

D.

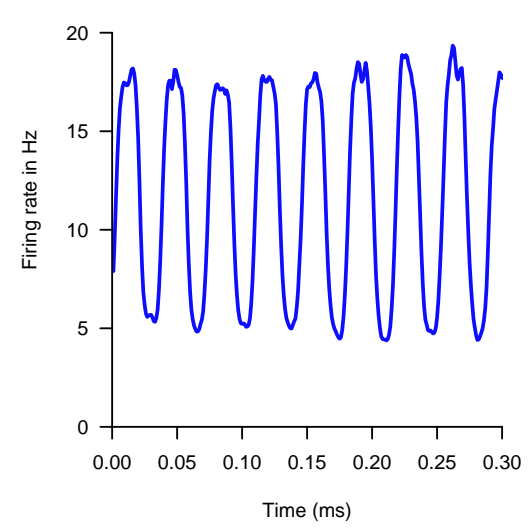

FSI

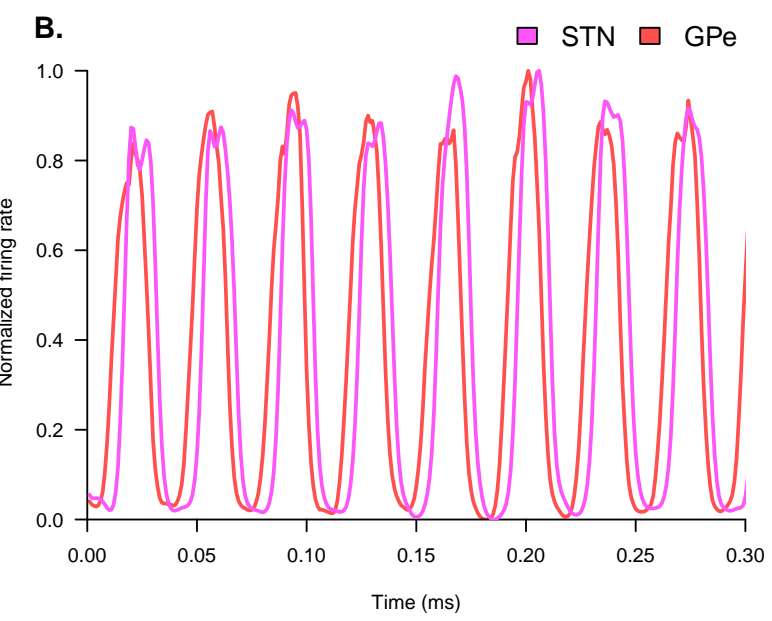

E.

GPi

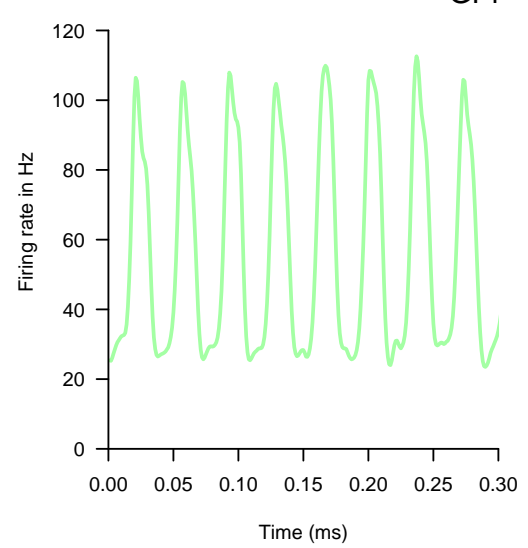

Figure 3: Synchronized oscillatory activity of STN and GPe caused by DA depletion. A. Relative STN-GPe firing rate traces obtained by simulation (grey) and their mean oscillatory pattern (blue). B. Sample of the normalized firing pattern of STN and GPe as a function of time. C. Sample activity of the other neural populations (MSN and FSI in the striatum, and GPi) during the same time interval. 


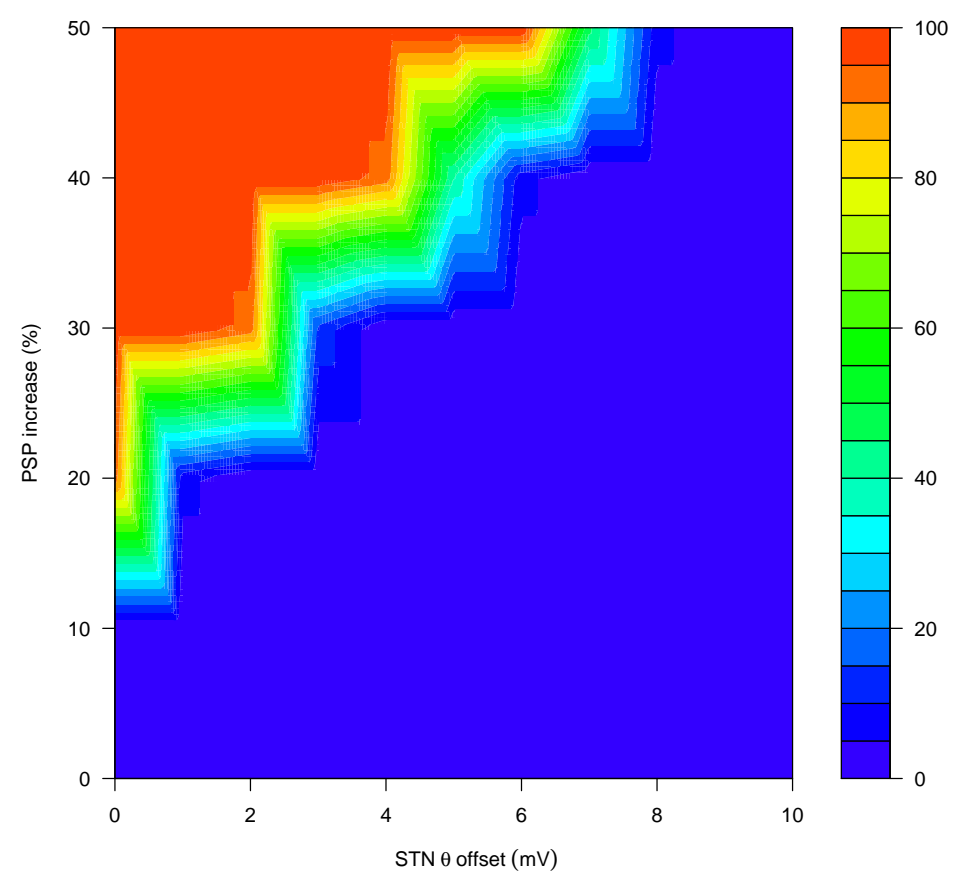

Figure 4: Proportion of the 15 model parameterizations exhibiting oscillations as a function of increasing post-synaptic potential (PSP) in GPe and STN (resulting from DA depletion on D2 receptors) and of increasing the activity threshold between resting and firing state in STN $(\theta$ offset, resulting from DA depletion on D5 receptors). The two latter factors control the emergence of oscillations in an antagonistic fashion. The boundary conditions to enter the oscillatory regime are an increase of PSP of at least 10\% in GPe and STN, and an increase of firing level threshold inferior to $8 \mathrm{mV}$ in the STN. 


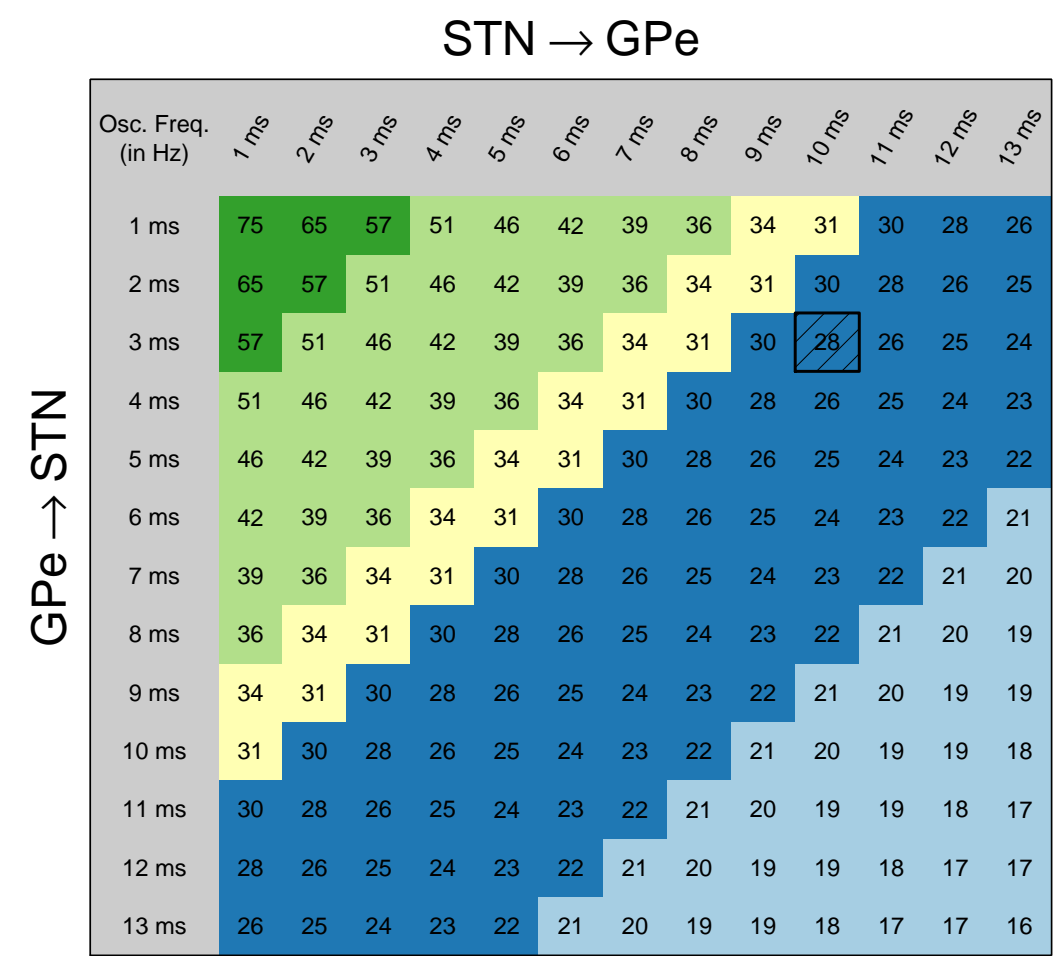

Figure 5: Frequency of oscillation (in $\mathrm{Hz}$ ) as a function of the axonal delays between STN and GPe. Delays from STN to GPe are listed as columns and from GPe to STN as rows. The set of most plausible axonal delays is shown in the dashed cell, and corresponds to STN $\rightarrow$ GPe delays of $10 \mathrm{~ms}$, and GPe $\rightarrow$ STN delays of 3 ms. Colors indicate different oscillation regimes, with the $\beta$-band $(13-30 \mathrm{~Hz})$ shown in blue and the $\gamma$-band $(35-80 \mathrm{~Hz})$ shown in green. These bands are further subdivided into lighter and darker hues indicating their lower/upper parts. No axonal delays close to these values can elicit $\alpha$-band oscillations in the extra-striate DA depletion model. 
2006, Tachibana et al., 2008, Iwamuro et al., 2009). To the best of our knowledge, this work is the first to perform a meta-analysis of these experimental studies by computational means. The main advantage of this approach over less formalized interpretation of stimulation studies (e.g. Jaeger and Kita, 2011) lies in its capacity to consider not only the average delays but also their confidence intervals. In addition, the rigorous approach presented here is reproducible and can readily be extended to new experimental studies. As a result, the delay estimates we obtained provide a full characterization of transmission properties within the basal ganglia (Table 1).

These delays lead to two testable predictions corresponding to empirical studies yet to be performed with macaque monkeys. To the best of our knowledge, no stimulation study has recorded the temporal time-course of: (a) the STN response following striatal stimulation, and (b) the STN response following a GPe stimulation. In case (a) we estimate that a striatal stimulation should yield an increase of activity in STN 17-19 ms later, through the Str $\rightarrow$ GPe $\rightarrow$ STN pathway (following the anatomical schema of Fig. S1). A subsequent inhibition should also follow through the STN/GPe rebound pathway $(\mathrm{Str} \rightarrow \mathrm{GPe} \rightarrow \mathrm{STN} \rightarrow$ $\mathrm{GPe} \rightarrow \mathrm{STN}$ ), 30-34 ms after the initial stimulation. In case (b) we estimate that the GPe stimulation should yield a decrease of activity after 10-11 ms (pathway GPe $\rightarrow$ STN), followed by an increase of activation after 23-26 ms (single rebound: GPe $\rightarrow$ STN $\rightarrow$ GPe $\rightarrow$ STN), and finally a decrease after 36-41 ms (double rebound: $\mathrm{GPe} \rightarrow \mathrm{STN} \rightarrow \mathrm{GPe} \rightarrow \mathrm{STN} \rightarrow \mathrm{GPe} \rightarrow \mathrm{STN}$, as in Fig. S3).

\section{Striatal and extra-striatal contributions to pathology}

Our results show that $\beta$-band oscillations arise in the BG circuitry when DA is depleted, irrespective of the effect of DA depletion onto DA receptors in the MSN and the FSI in the Striatum, which we disregarded in our model. This suggests that the direct/indirect pathway segregation may not be central in the understanding of the PD oscillatory phenomena. It nevertheless remains to be addressed whether and to what extent does the Striatum contribute to such oscillations.

By removing our focus from the segregation of striatal D1/D2 receptors, we implicitly assumed that the excitatory and inhibitory effects of DA in the striatum cancel each other, resulting in an average null effect on MSN firing rate. Consistently with this, electro-physiological studies in primates reported no change in the striatal firing rate after MPTP injection (Goldberg et al., 2002). Furthermore, the modification of the distribution of the firing rates in the MSN population (decreased activity in D1 neurons, increased activity in D2 ones) would exert little influence on the STN $\leftrightarrow$ GPe loop, as a consequence of the massively collateralized striato-fugal projection in primates (Parent et al., 1995, Lévesque and Parent, 2005, Nadjar et al., 2006).

Finally, we did not model the effect of DA depletion on the GPi, first, because experimental data on the effects of such depletion remain incomplete (Rommelfanger and Wichmann, 2010), and second, because the GPi does not project inside the modeled BG circuitry, and thus cannot participate of the local generation of oscillations. Should the model be extended to encompass the cortico-baso-thalamo loops, the GPi would become a central actor of these loops, and as such would participate actively in their dynamics. Modeling GPi DA receptor would then become essential to determine the effects of DA depletion on the whole circuit.

\section{Sources of $\beta$-band oscillations}

We discuss next a list of most prominent theories regarding the origin of $\beta$-band oscillations proposed by previous computational studies. First, the striatal origin theory suggests that intrinsic properties of the MSNs alone are the key to generate oscillatory behaviour in the basal ganglia McCarthy et al. (2011). However, this is at odds with experimental data showing that suppressing the inhibitory inputs from the MSN to the GPe did not cancel the GPe oscillatory activity in monkey's model of PD (Tachibana et al., 2011), as clearly argued by Pavlides et al. (2015). Second, the striatal inhibition theory has been introduced by two recent computational studies, suggesting that the increased striatal inhibition by means of the D2 neurons on the GPe-STN loop (Kumar et al., 2011, Lindahl and Hellgren Kotaleski, 2016) would cause the pathological oscillations. However, this is at odds with the aforementioned experiment by Tachibana et al. (2011) and relies on the segregation of striatofugal pathways. Third, the often overlooked GPe to Striatum feedback projection 
(typically to the FSI), has also been proposed (Corbit et al., 2016) to be an essential component of the generation of $\beta$-band oscillations via the MSN-GPe-FSI loop (FSI loop theory). This theory is in contradiction with the experimental results of Tachibana et al. (2011), and our simulation study shows that this loop would instead act as relay transferring the GPe-STN oscillations back to the striatum (Figs. S5 and S6). Fourth, the (cortico-basal theory) suggested that $\beta$-band oscillations originate in the thalamo-cortical loop, and are then amplified in the BG (Van Albada and Robinson, 2009, Van Albada et al., 2009). However, the Tachibana et al. (2011) study showed that suppressing the GPe inhibition to the STN suppressed oscillations, thus ruling out cortical inputs as the primary oscillation cause. Also, this theory requires segregated indirect/D2 pathways, which is again at odds with primate neuro-anatomy. Leblois et al. (2006a) examined under which conditions the long cortico-baso-thalamo-cortical loop could be the source of $\beta$-oscillations. While such a circuit could indeed sustain oscillatory activity, the long delays involved to run through the entire loop makes it more suitable to slower rhythms, such as $\theta$-band oscillations that may be linked to PD. Finally, Pavlides et al. (2015) have shown that it is possible to parameterize a simplified cortico-basal model so that three loops (the STN $\leftrightarrow$ GPe one, the intra-cortical one and the long cortico-basal one) contribute to the emergence of $\beta$ oscillations, without any of them being able to autonomously sustain them (distributed sources theory). This model has been optimized to model the pathological state only, using electrophysiology data alone. Our model, which encompasses the whole BG circuitry, and which is constrained by both anatomical and physiological data of healthy subjects, shows that the STN $\leftrightarrow$ GPe projection under normal conditions is ready to oscillate autonomously in the $\beta$-band, and does so as soon as the coupling between STN and GPe is increased.

Early models (Gillies et al., 2002, Terman et al., 2002) suggesting that the GPe-STN loop could be the generator of abnormal $\beta$ oscillations in PD (GPe-STN loop theory), neglected transmission delays between the GPe and the STN and instead relied on an input imbalance to this loop to elicit oscillations. We have shown here that the transmission delays play a crucial role in setting the oscillation frequency, in addition to the neural intrinsic dynamic processes. Moreover, we have also shown, with synaptic strength and delay parameters derived from experimental data, that extrastriate D2 receptors which affect the coupling between the GPe and the STN are sufficient to generate the PD oscillations, so that striatal or cortical activity modifications are not necessary. The model proposed by Humphries et al. (2006) is based on rodent data and exhibits $\gamma$-band as well as slow $(<1 \mathrm{~Hz})$ oscillations, consistently with experimental data in this species. Interestingly, when using the axonal delays compiled from rodent literature by Humphries et al. (specifically, $4 \mathrm{~ms}$ for the rodent equivalent of $\mathrm{GPe} \rightarrow \mathrm{STN}$ and $2 \mathrm{~ms}$ for STN $\rightarrow \mathrm{GPe}$ ), our primate model predicts oscillations at $46 \mathrm{~Hz}$ (Fig. 5), a value relatively close to the oscillation frequency of the rodent model (53-55 $\mathrm{Hz}$ ). This suggests that high-frequency $\gamma$-band oscillations in rats could be equivalent to $\beta$-band oscillations in primates, set in a higher band because of shorter transmission delays in the GPe $\leftrightarrow$ STN loop.

In their model restricted to the GPe $\leftrightarrow$ STN loop, Nevado Holgado et al. (2010) stressed on the importance of the transmission delays in this loop to set the oscillation frequency. They parameterized the model with a limited set of electrophysiological constraints (not including pharmacological receptor deactivations) and no anatomical ones. As a result, their model requires surprisingly strong increases in synaptic strengths (two-fold increase for GPe $\rightarrow$ GPe, almost four-fold for cortex $\rightarrow$ STN, almost ten-fold for striatum $\rightarrow$ GPe and GPe $\rightarrow$ STN). The increased striatum $\rightarrow$ GPe projection shows that a segregated indirect/D2 pathway is also necessary. We could obtain oscillations with less than 1.5-fold increases of the STN $\rightarrow$ GPe and $\mathrm{GPe} \rightarrow$ STN connection strengths only, and without pathway segregation.

The Tsirogiannis et al. (2010) model also advocates for the GPe-STN loop theory, although it requires segregated direct/indirect pathway, uses unrealistically short transmission delays (1 ms for both GPe $\rightarrow$ STN and STN $\rightarrow$ GPe connections, cf. Table 1) and requires that PD affects both the amplitude and time-course of post-synaptic potentials. We managed to exhibit such oscillations without segregated pathways, with transmission delays compatible with experimental stimulation results, and with a more parsimonious model of D2 receptors reaction to lack of dopamine (the modulation of the amplitude of the post-synaptic potential being sufficient).

Shouno et al. (2017) recently proposed a spiking model of the GPe $\leftrightarrow$ STN loop in primates. They managed to simulate the main tendencies of the experimental results of Tachibana et al. (2011). In their 
model, the post-inhibitory rebound excitation at the STN level and short-term plasticity in both STN and GPe are essential to trigger oscillations. While these properties may indeed contribute to the phenomenon, and certainly alter the oscillation frequencies, we have shown with a more parsimonious model that the emergence of oscillations does not require these properties.

The main implication of this study is to support the hypothesis that $\beta$ oscillations are generated by the STN to GPe coupling when there is DA loss. In this and our previous work (Liénard and Girard, 2014), we proposed a model of the basal ganglia that is entirely data-based, in the sense that all connection delays and strengths were optimized to fit to experimental data. The main contribution of this study is to establish that $\beta$-band oscillations characteristic of PD could be generated by DA depletion involving the GPe and the STN alone. Here we have also shown that that GPe/STN synchronous activity can be propagated to the FSI-MSN circuit. Also, in our model the frequency of oscillations is conditioned on the connection delays within the GPe $\leftrightarrow$ STN loop. Shorter delays would lead to oscillations with higher frequencies, while the slower $\theta$-band (4-8Hz) and $\alpha$-band $(8-12 \mathrm{~Hz})$ oscillations in the STN $\leftrightarrow$ GPe loop would require non plausible long delays in their interconnections (Fig. 5). An interesting consequence is thus that the slower $\theta$ and $\alpha$ oscillations cannot be generated in the GPe $\leftrightarrow$ STN loop with the level of detail of our model. By elimination, these slower oscillations should then originate in a loop involving the cortex and the thalamus.

\section{Acknowledgements}

This project was partially funded by the ANR EvoNeuro project, ANR-09-EMER-005-01, as well as by the Laboratory of Excellence SMART (ANR-11-LABX-65), supported by French State funds managed by the ANR within the Investissements d'Avenir programme under reference ANR-11-IDEX-0004-02. IC was funded by the Ville de Paris HABOT Project, and by the EU Horizon 2020 Research and Innovation program, Marie Curie IF No. 656262.

\section{References}

Albin, R., Young, A., and Penney, J. (1989). The functional anatomy of basal ganglia disorders. Trends in Neurosciences, 12(10):366-375.

Bauswein, E., Fromm, C., and Preuss, A. (1989). Corticostriatal cells in comparison with pyramidal tract neurons: contrasting properties in the behaving monkey. Brain Research, 493(1):198-203.

Bellinger, S., Miyazawa, G., and Steinmetz, P. (2008). Submyelin potassium accumulation may functionally block subsets of local axons during deep brain stimulation: a modeling study. Journal of neural engineering, $5(3): 263$.

Benazzouz, A., Mamad, O., Abedi, P., Bouali-Benazzouz, R., and Chetrit, J. (2014). Involvement of dopamine loss in extrastriatal basal ganglia nuclei in the pathophysiology of parkinson's disease. Frontiers in aging neuroscience, 6 .

Berardelli, A., Hallett, M., Rothwell, J. C., Agostino, R., Manfredi, M., Thompson, P. D., and Marsden, C. D. (1996). Single-joint rapid arm movements in normal subjects and in patients with motor disorders. Brain, 119(2):661-674.

Berardelli, A., Rothwell, J. C., Thompson, P. D., and Hallett, M. (2001). Pathophysiology of bradykinesia in Parkinson's disease. Brain, 124(11):2131-2146.

Brown, P. and Marsden, C. D. (1999). Bradykinesia and impairment of eeg desynchronization in parkinson's disease. Movement disorders, 14(3):423-429. 
Brown, P., Oliviero, A., Mazzone, P., Insola, A., Tonali, P., and Di Lazzaro, V. (2001). Dopamine dependency of oscillations between subthalamic nucleus and pallidum in parkinson's disease. The Journal of Neuroscience, $21(3): 1033-1038$.

Cazorla, M., de Carvalho, F. D., Chohan, M. O., Shegda, M., Chuhma, N., Rayport, S., Ahmari, S. E., Moore, H., and Kellendonk, C. (2014). Dopamine d2 receptors regulate the anatomical and functional balance of basal ganglia circuitry. Neuron, 81(1):153-164.

Chetrit, J., Taupignon, A., Froux, L., Morin, S., Bouali-Benazzouz, R., Naudet, F., Kadiri, N., Gross, C. E., Bioulac, B., and Benazzouz, A. (2013). Inhibiting subthalamic d5 receptor constitutive activity alleviates abnormal electrical activity and reverses motor impairment in a rat model of parkinson's disease. The Journal of Neuroscience, 33(37):14840-14849.

Corbit, V. L., Whalen, T. C., Zitelli, K. T., Crilly, S. Y., Rubin, J. E., and Gittis, A. H. (2016). Pallidostriatal projections promote $\beta$ oscillations in a dopamine-depleted biophysical network model. Journal of Neuroscience, 36(20):5556-5571.

Debanne, D., Campanac, E., Bialowas, A., Carlier, E., and Alcaraz, G. (2011). Axon physiology. Physiological Reviews, 91(2):555-602.

Doyle, L., Kühn, A., Hariz, M., Kupsch, A., Schneider, G.-H., and Brown, P. (2005). Levodopa-induced modulation of subthalamic beta oscillations during self-paced movements in patients with parkinson's disease. European Journal of Neuroscience, 21(5):1403-1412.

Filion, M. and Tremblay, L. (1991). Effects of dopamine agonists on the spontaneous activity of globus pallidus neurons in monkeys with mptp-induced parkinsonism. Brain Research, 547(1):145-149.

Frank, M. (2005). Dynamic dopamine modulation in the basal ganglia: a neurocomputational account of cognitive deficits in medicated and nonmedicated parkinsonism. Journal of Cognitive Neuroscience, 17(1):51-72.

Frank, M. J., Seeberger, L. C., and O'reilly, R. C. (2004). By carrot or by stick: cognitive reinforcement learning in parkinsonism. Science, 306(5703):1940-1943.

Fujiyama, F., Sohn, J., Nakano, T., Furuta, T., Nakamura, K., Matsuda, W., and Kaneko, T. (2011). Exclusive and common targets of neostriatofugal projections of rat striosome neurons: a single neuron-tracing study using a viral vector. European Journal of Neuroscience, 33(4):668-677.

Gillies, A., Willshaw, D., and Li, Z. (2002). Subthalamic-pallidal interactions are critical in determining normal and abnormal functioning of the basal ganglia. Proceedings: Biological Sciences, pages 545-551.

Goldberg, J., Boraud, T., Maraton, S., Haber, S., Vaadia, E., and Bergman, H. (2002). Enhanced synchrony among primary motor cortex neurons in the 1-methyl-4-phenyl-1, 2, 3, 6-tetrahydropyridine primate model of parkinson's disease. The Journal of Neuroscience, 22(11):4639-4653.

Gurney, K., Prescott, T., and Redgrave, P. (2001). A computational model of action selection in the basal ganglia. ii. analysis and simulation of behaviour. Biological Cybernetics, 84(6):411-423.

Holgado, A., Terry, J., and Bogacz, R. (2010). Conditions for the generation of beta oscillations in the subthalamic nucleus-globus pallidus network. The Journal of Neuroscience, 30(37):12340-12352.

Humphries, M., Stewart, R., and Gurney, K. (2006). A physiologically plausible model of action selection and oscillatory activity in the basal ganglia. The Journal of Neuroscience, 26(50):12921.

Iwamuro, H., Tachibana, Y., Saito, N., and Nambu, A. (2009). Organization of motor cortical inputs to the subthalamic nucleus in the monkey. The Basal Ganglia IX, pages 109-117. 
Jaeger, D. and Kita, H. (2011). Functional connectivity and integrative properties of globus pallidus neurons. Neuroscience, 198:44-53.

Kawaguchi, Y., Wilson, C., and Emson, P. (1990). Projection subtypes of rat neostriatal matrix cells revealed by intracellular injection of biocytin. The Journal of Neuroscience, 10(10):3421-3438.

Kita, H., Chiken, S., Tachibana, Y., and Nambu, A. (2006). Origins of gabaa and gabab receptor-mediated responses of globus pallidus induced after stimulation of the putamen in the monkey. The Journal of Neuroscience, 26(24):6554-6562.

Kita, H., Nambu, A., Kaneda, K., Tachibana, Y., and Takada, M. (2004). Role of ionotropic glutamatergic and gabaergic inputs on the firing activity of neurons in the external pallidum in awake monkeys. Journal of Neurophysiology, 92(5):3069-3084.

Kita, H., Tachibana, Y., Nambu, A., and Chiken, S. (2005). Balance of monosynaptic excitatory and disynaptic inhibitory responses of the globus pallidus induced after stimulation of the subthalamic nucleus in the monkey. The Journal of Neuroscience, 25(38):8611-8619.

Koshimizu, Y., Fujiyama, F., Nakamura, K. C., Furuta, T., and Kaneko, T. (2013). Quantitative analysis of axon bouton distribution of subthalamic nucleus neurons in the rat by single neuron visualization with a viral vector. Journal of Comparative Neurology, 521(9):2125-2146.

Kühn, A. A., Kupsch, A., Schneider, G.-H., and Brown, P. (2006). Reduction in subthalamic 8-35 hz oscillatory activity correlates with clinical improvement in parkinson's disease. European Journal of Neuroscience, 23(7):1956-1960.

Kumar, A., Cardanobile, S., Rotter, S., and Aertsen, A. (2011). The role of inhibition in generating and controlling parkinson's disease oscillations in the basal ganglia. Frontiers in Systems Neuroscience, 5.

Leblois, A., Boraud, T., Meissner, W., Bergman, H., and Hansel, D. (2006a). Competition between feedback loops underlies normal and pathological dynamics in the basal ganglia. The Journal of neuroscience, 26(13):3567-3583.

Leblois, A., Boraud, T., Meissner, W., Bergman, H., and Hansel, D. (2006b). Competition between feedback loops underlies normal and pathological dynamics in the basal ganglia. The Journal of Neuroscience, 26(13):3567-3583.

Lévesque, M. and Parent, A. (2005). The striatofugal fiber system in primates: a reevaluation of its organization based on single-axon tracing studies. Proceedings of the National Academy of Sciences, 102(33):11888-11893.

Levy, R., Hutchison, W. D., Lozano, A. M., and Dostrovsky, J. O. (2000). High-frequency synchronization of neuronal activity in the subthalamic nucleus of parkinsonian patients with limb tremor. The Journal of neuroscience, 20(20):7766-7775.

Liénard, J. and Girard, B. (2014). A biologically constrained model of the whole basal ganglia addressing the paradoxes of connections and selection. Journal of Computational Neuroscience, 36(3):445-468.

Lindahl, M. and Hellgren Kotaleski, J. (2016). Untangling basal ganglia network dynamics and function: Role of dopamine depletion and inhibition investigated in a spiking network model. eNeuro, 3(6):e0156-16.2016.

Marsden, C. D. (1984). Which motor disorder in Parkinson's disease indicates the true motor function of the basal ganglia? Ciba Foundation symposium, 107.

Marsden, C. D. (1989). Slowness of movement in Parkinson's disease. Movement Disorders: Official Journal of the Movement Disorder Society, 4 Suppl 1:S26-37. 
Mazzoni, P., Shabbott, B., and Cortés, J. C. (2012). Motor abnormalities in parkinson's disease. Cold Spring Harbor Perspectives in Medicine, 2.

McCarthy, M., Moore-Kochlacs, C., Gu, X., Boyden, E., Han, X., and Kopell, N. (2011). Striatal origin of the pathologic beta oscillations in parkinson's disease. Proceedings of the National Academy of Sciences, 108(28):11620-11625.

Nadjar, A., Brotchie, J., Guigoni, C., Li, Q., Zhou, S., Wang, G., Ravenscroft, P., Georges, F., Crossman, A., and Bezard, E. (2006). Phenotype of striatofugal medium spiny neurons in parkinsonian and dyskinetic nonhuman primates: a call for a reappraisal of the functional organization of the basal ganglia. The Journal of Neuroscience, 26(34):8653-8661.

Nambu, A., Kaneda, K., Tokuno, H., and Takada, M. (2002). Organization of corticostriatal motor inputs in monkey putamen. Journal of Neurophysiology, 88(4):1830-1842.

Nambu, A., Tokuno, H., Hamada, I., Kita, H., Imanishi, M., Akazawa, T., Ikeuchi, Y., and Hasegawa, N. (2000). Excitatory cortical inputs to pallidal neurons via the subthalamic nucleus in the monkey. Journal of Neurophysiology, 84(1):289-300.

Nevado Holgado, A., Terry, J., and Bogacz, R. (2010). Conditions for the generation of beta oscillations in the subthalamic nucleus-globus pallidus network. The Journal of Neuroscience, 30(37):12340-12352.

Nini, A., Feingold, A., Slovin, H., and Bergman, H. (1995). Neurons in the globus pallidus do not show correlated activity in the normal monkey, but phase-locked oscillations appear in the mptp model of parkinsonism. Journal of Neurophysiology, 74(4):1800-1805.

Oswal, A., Brown, P., and Litvak, V. (2013). Synchronized neural oscillations and the pathophysiology of parkinson's disease. Current opinion in neurology, 26(6):662-670.

Parent, A., Charara, A., and Pinault, D. (1995). Single striatofugal axons arborizing in both pallidal segments and in the substantia nigra in primates. Brain research, 698(1):280-284.

Pasquereau, B., DeLong, M. R., and Turner, R. S. (2015). Primary motor cortex of the parkinsonian monkey: altered encoding of active movement. Brain, page awv312.

Pasquereau, B. and Turner, R. (2011). Primary motor cortex of the parkinsonian monkey: Differential effects on the spontaneous activity of pyramidal tract-type neurons. Cerebral Cortex, 21(6):1362-1378.

Pavlides, A., Hogan, S. J., and Bogacz, R. (2015). Computational models describing possible mechanisms for generation of excessive beta oscillations in parkinson's disease. PLoS Comput Biol, 11(12):e1004609.

Rommelfanger, K. S. and Wichmann, T. (2010). Extrastriatal dopaminergic circuits of the basal ganglia. Frontiers in neuroanatomy, 4.

Samii, A., Nutt, J. G., and Ransom, B. R. (2004). Parkinson's disease. The Lancet, 363(9423):1783-1793.

Shouno, O., Tachibana, Y., Nambu, A., and Doya, K. (2017). Computational model of recurrent subthalamopallidal circuit for generation of parkinsonian oscillations. Frontiers in Neuroanatomy, 11(21).

Tachibana, Y., Iwamuro, H., Kita, H., Takada, M., and Nambu, A. (2011). Subthalamo-pallidal interactions underlying parkinsonian neuronal oscillations in the primate basal ganglia. European Journal of Neuroscience, $34(9): 1470-1484$.

Tachibana, Y., Kita, H., Chiken, S., Takada, M., and Nambu, A. (2008). Motor cortical control of internal pallidal activity through glutamatergic and gabaergic inputs in awake monkeys. European Journal of Neuroscience, 27(1):238-253. 
Terman, D., Rubin, J., Yew, A., and Wilson, C. (2002). Activity patterns in a model for the subthalamopallidal network of the basal ganglia. The Journal of Neuroscience, 22(7):2963-2976.

Tsirogiannis, G., Tagaris, G., Sakas, D., and Nikita, K. (2010). A population level computational model of the basal ganglia that generates parkinsonian local field potential activity. Biological Cybernetics, 102(2):155-176.

Turner, R. and DeLong, M. (2000). Corticostriatal activity in primary motor cortex of the macaque. The Journal of Neuroscience, 20(18):7096-7108.

Valjent, E., Bertran-Gonzalez, J., Hervé, D., Fisone, G., and Girault, J.-A. (2009). Looking bac at striatal signaling: cell-specific analysis in new transgenic mice. Trends in neurosciences, 32(10):538-547.

Van Albada, S., Gray, R., Drysdale, P., Robinson, P., et al. (2009). Mean-field modeling of the basal ganglia-thalamocortical system. II Dynamics of parkinsonian oscillations. Journal of Theoretical Biology, 257(4):664-688.

Van Albada, S. and Robinson, P. (2009). Mean-field modeling of the basal ganglia-thalamocortical system. I Firing rates in healthy and parkinsonian states. Journal of Theoretical Biology, 257(4):642-663.

Weinberger, M., Hutchison, W. D., and Dostrovsky, J. O. (2009). Pathological subthalamic nucleus oscillations in pd: can they be the cause of bradykinesia and akinesia? Experimental neurology, 219(1):58-61.

Wu, Y., Richard, S., and Parent, A. (2000). The organization of the striatal output system: a single-cell juxtacellular labeling study in the rat. Neuroscience Research, 38(1):49-62.

Yelnik, J. and Percheron, G. (1979). Subthalamic neurons in primates: a quantitative and comparative analysis. Neuroscience, 4(11):1717-1743.

Yoshida, S., Nambu, A., and Jinnai, K. (1993). The distribution of the globus pallidus neurons with input from various cortical areas in the monkeys. Brain Research, 611(1):170-174. 